\title{
2021 consensus statement for preventing and managing low back pain in elite and subelite adult rowers
}

\author{
Fiona Wilson (D) , Jane S Thornton (D) , ${ }^{2,3}$ Kellie Wilkie, ${ }^{4}$ Jan Hartvigsen, ${ }^{5}$ \\ Anders Vinther, ${ }^{6}$ Kathryn E Ackerman (D) , 7 P Caneiro, ${ }^{8}$ Larissa Trease (D) , \\ Frank Nugent, ${ }^{10}$ Conor Gissane, ${ }^{1}$ Sarah-Jane McDonnell, ${ }^{11}$ Alison McGregor, ${ }^{12}$ \\ Craig Newlands, ${ }^{13}$ Clare LArdern (D) ${ }^{14,15}$
}

\begin{abstract}
- Additional material is published online only. To view, please visit the journal online (http://dx.doi.org/10.1136/ bjsports-2020-103385).
\end{abstract}

For numbered affiliations see end of article.

Correspondence to Fiona Wilson, Discipline of Physiotherapy, Trinity College Dublin School of Medicine, Dublin 2, Ireland; wilsonf@tcd.ie

Accepted 19 February 2021 Published Online First 8 March 2021

\section{ABSTRACT \\ Purpose To synthesise evidence on low back pain (LBP) in adult rowers and to create a consensus statement to inform clinical practice.}

Methods There were four synthesis steps that informed the consensus statement. In step one, seven expert clinicians and researchers established the scope of the consensus statement and conducted a survey of experienced and expert clinicians to explore current practice. In step two, working groups examined current evidence relating to key scope questions and summarised key issues. In step three, we synthesised evidence for each group and used a modified Delphi process to aid in the creation of the overall consensus statements. Finally, in step four, we combined information from step three with the findings of the clinician survey (and with athlete and coach input) to produce recommendations for clinical practice.

Results The scope of the consensus statement included epidemiology; biomechanics; management; the athlete's voice and clinical expertise. Prevention and management of LBP in rowers should include education on risk factors, rowing biomechanics and training load. If treatment is needed, non-invasive management, including early unloading from aggravating activities, effective pain control and exercise therapy should be considered. Fitness should be maintained with load management and progression to full training and competition. The role of surgery is unclear. Management should be athlete focused and a culture of openness within the team encouraged.

Conclusion Recommendations are based on current evidence and consensus and aligned with international LBP guidelines in non-athletic populations, but with advice aimed specifically at rowers. We recommend that research in relation to all aspects of prevention and management of LBP in rowers be intensified.

\section{INTRODUCTION}

Low back pain (LBP) is the most frequently reported musculoskeletal disorder in the community, and can result in long-term pain and disability. ${ }^{12}$ Rowing is a sport associated with large volumes of training and high cumulative loading of the lumbar spine. The most frequently reported site of pain for rowers, as a result of rowing, is also the low back. ${ }^{34}$ The most recently published prospective study reported that $21 \%$ of all reported illness and injuries over eight seasons in a national rowing team were to the lumbar spine. ${ }^{5}$ Recent research has focused on epidemiology and biomechanical analyses to understand mechanisms that contribute to LBP onset. There has been a limited focus on management or prevention strategies.

There are currently no guidelines for managing LBP in rowers (hereby defined as 'rowing-related LBP') or in athletes who participate in other sports. There are guidelines for managing LBP in the general population, however while some principles of management are transferable, there is a need to consider issues that are particular to rowers and athletes.

The overall aim of this project was to inform clinical decisions and standards of care in order to reduce the long-term effects of LBP on rowers, and to influence outcome by reducing personal burden and healthcare costs.

Specifically, we aim to (1) synthesise and present the current evidence on LBP in adult rowers and (2) develop practical recommendations for prevention and management to facilitate translating evidence into practice.

\section{METHODS}

The AGREE II reporting checklist ${ }^{6}$ (www.agreetrust.org) guided development and reporting of this consensus statement.

Figure 1 summarises the consensus statement methods.

\section{Contributors}

A core expert group comprising seven individuals (FW, JST, KW, AV, AMcG, CG and CN) who had published research in rowing-related LBP and who had broad experience of managing rowers with LBP, convened at British Rowing Headquarters, Hammersmith, London, in February 2018. The group defined the objectives of the consensus statement and outlined the personnel required. Two methods experts (JH and CLA) were invited and consulted throughout the process of developing the consensus statement and recommendations. The Medical Commission of the international rowing body 'World Rowing' oversaw and supported the process. World Rowing did not provide any financial support, nor did they have any editorial input, but they have endorsed this document. 


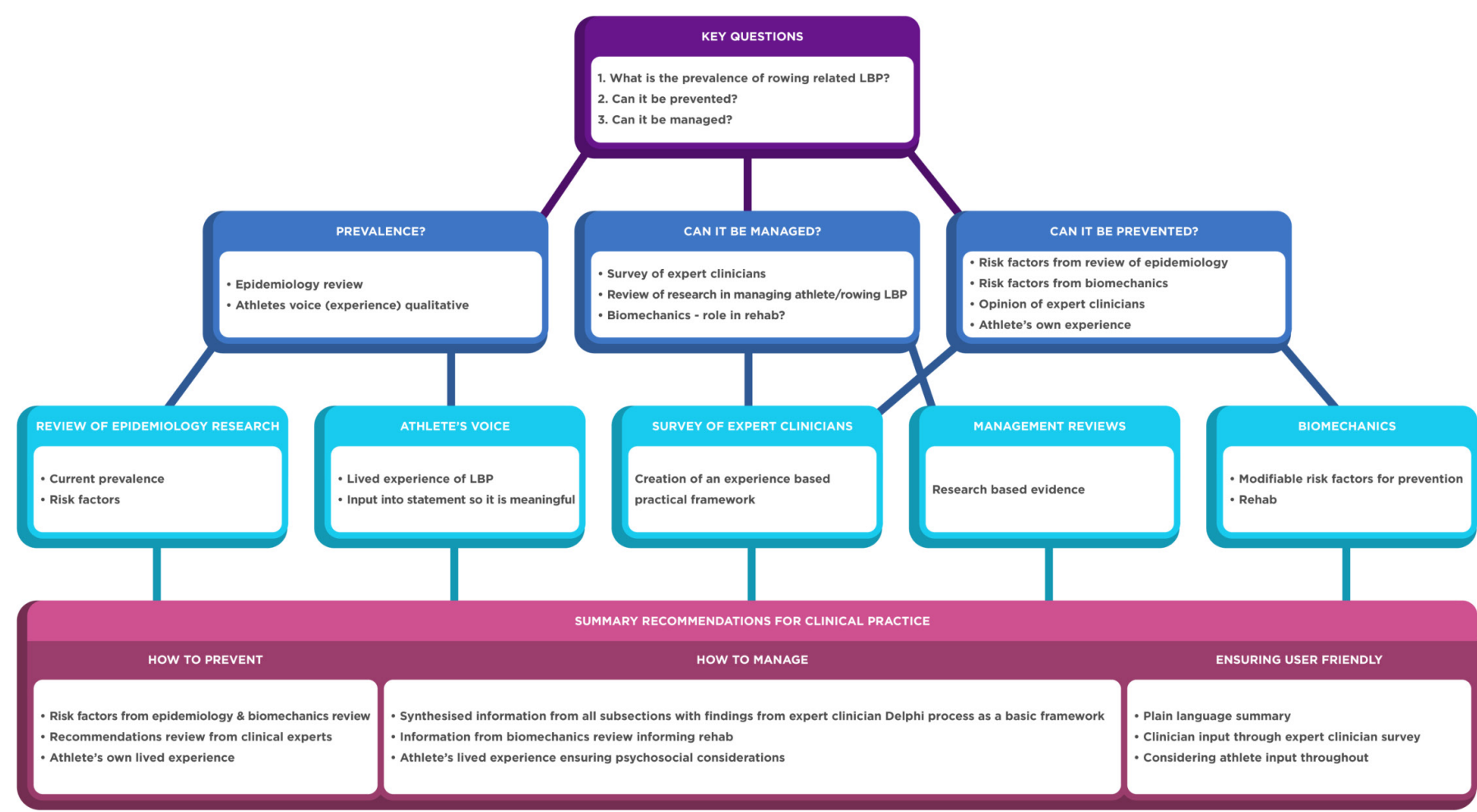

Figure 1 Summary of consensus statement methods.

Following the February 2018 meeting, additional experts were identified for each subsection based on relevant expertise. Experts were required to have (i) been engaged in managing rowing-related LBP, and (ii) conducted research in the area, which they had disseminated. The expert group included physiotherapists, physicians (sports medicine and endocrinology), orthopaedic surgeons, coaches, sport scientists (eg, strength and conditioning experts) and athlete representatives. The group represented all key end users and reflected the composition of the appropriate World Rowing Commissions and the range of key characteristics of the user populations.

Expert group members represented Europe, North America and Australasia, which comprise the greatest number of rowing nations. All rowing nations had the opportunity to contribute to the consensus statement via (i) a clinician survey distributed to every nation registered with World Rowing, and (ii) at an interim presentation of the protocol and preliminary results at the World Rowing Sports Medicine, Science and Coaches Conference in Berlin, November 2018.

\section{Setting the task and defining questions}

The keynote presentation from the 2015 World Rowing Championships (WRC) Medical Meeting was used as the reference document when defining the scope of this consensus statement. ${ }^{7}$ This presentation had synthesised and summarised the evidence on rowing-related LBP, and was later posted on the World Rowing website in $2016^{7}$ to inform and invite discussion by the world rowing community. The content of the community feedback on the website, input from the World Rowing Sports Medicine Commission members and informal feedback (audience questions and debate) from the WRC meeting were considered and used to inform discussion at the February 2018 meeting in London.

The first task of the February 2018 meeting was to define the questions that would underpin the consensus statement. Three key questions were discussed in a round-table format at the London meeting:

1. What is the extent (prevalence) of rowing-related LBP; how does it compare to other sports and LBP in the general population?

2. Can rowing-related LBP be managed, and how?

3. Can rowing-related LBP be prevented?

\section{Consensus objectives}

The core expert group defined project objectives by discussing the key questions; reflecting on how established research could answer these and where new research was required.

We aimed to answer questions 1 and 2 by reviewing epidemiology and management strategies. To understand management, we planned a systematic review to evaluate the evidence for non-pharmacological management of LBP in athletes. ${ }^{8}$ We then planned an extensive survey of expert and experienced clinicians to investigate current best clinical assessment and management of an acute episode of LBP in rowers. ${ }^{9}$ In addition, we planned qualitative research to investigate the athlete's lived experience. ${ }^{10}$

We agreed that the focus of question 3 would be to explore risk factors reported in epidemiology and to examine the influence of biomechanics as a modifiable and influencing factor in rowing-related LBP. Initial discussions and informal scoping of rowing-related LBP studies identified a considerable research focus on biomechanics, and we agreed to create a subgroup to explore the research on rowing biomechanics. ${ }^{11}$ As part of the clinician survey, we also asked clinicians for their opinions on modifiable risk factors.

The following set of objectives were agreed by consensus (box 1).

To accomplish our objectives, we established working groups. Two groups were charged with conducting original research: a qualitative study of the rowers' lived experience of LBP, and a Delphi survey of rowing clinicians' opinions. One group had collected data prior to 


\section{Box 1 Objectives of London meeting, February 2018}

\section{Objectives}

- Perform systematic reviews of epidemiology, biomechanics and management of low back pain.

- To seek information from end users.

- Rowing athletes to examine their experiences of low back pain.

- Clinicians to investigate their opinions, experiences and recommendations regarding rowing-related low back pain management.

- Provide a definition of rowing-related low back pain.

- To create a framework for managing rowing-related low back pain with recommendations regarding recognising, triaging and managing pain in the acute, subacute and chronic phases.

- Develop recommendations for rehabilitation and prevention advice

the February 2018 meeting (the qualitative study), so were reconvened for the consensus statement project.

Three groups were established to conduct systematic reviews. The first group was charged with framing a definition of rowing-related LBP and conducting a systematic review of literature on epidemiology of LBP in athletes, with a subgroup analysis of studies that examined rowing-related $\mathrm{LBP}^{12}$ The second group examined the biomechanics associated with rowing-related $\mathrm{LBP}^{11}$ and the third reviewed the treatment of LBP in athletes with studies examining rowing-related LBP synthesised where possible. ${ }^{8}$

The full methodologies and outputs from each work group are presented as companion papers to this consensus statement. ${ }^{8-12}$ The study proposal, interim findings and key questions (from the February 2018 meeting) were presented at the World Rowing Sports Medicine, Science and Coaches Conference in Berlin, November 2018.

\section{Modified Delphi process to decide on the content of the consensus statement}

For each of the outputs from the working groups and on completion and analysis of findings, a series of summary statements and recommendations (where possible) were created to reflect the study or review findings. We used a modified two-to-four round Delphi process.

Ahead of round one, we used content analysis to summarise key results using Microsoft Excel (Microsoft Corporation, USA) and Google Docs (www.docs.google.com) for online sharing with each work group. Members of the work groups anonymously rated their level of agreement on a 10-point scale where $1=$ disagree strongly and $10=$ agree strongly. Respondents could add statements or suggest modifications to the original statements. Agreement was established when the mean reached a score of seven or above as a representation of combined group opinion.

For round two, the agreed statements, and those that were added in round one, were shared with each working group and the core expert group, along with a completed copy of the study findings. Voting was again conducted anonymously in the Google Doc as described, and the group was invited to add or modify the statements. These invited comments and modifications were also voted on at this stage. In round three, participants rerated their level of agreement for each statement after viewing scoring distribution of group opinion from round two. Consensus for a statement was established when the round three mean score reached seven or above and the SD was two or less. If necessary, a fourth round was conducted. For example, if any authors added modifications of any of the agreed statements at round three.

A separate voting process was conducted by the epidemiology group for the definition of LBP where experts who had published in athlete LBP were also invited to vote in an initial three-round process. A fourth-round vote was conducted and the final statement that was chosen was the one with the highest mean score.

The survey of experienced and expert clinicians was conducted from the outset as a separate Delphi process and methods are detailed in the accompanying paper. ${ }^{9}$

The final summaries and recommendations were based on assessing the quality of evidence, patient values and preferences as well as the experience and insight in the work groups.

\section{Plain language summary}

On completion of the consensus statement, three athletes (GO'D, FS and $\mathrm{KB}$ ) and two coaches (MH and PT) independently provided feedback on content and language. A plain language summary was constructed using their feedback and was guided by methods outlined by the Cochrane Collaboration ${ }^{13}$ (online supplemental file 6)

\section{RESULTS}

\section{Findings of systematic reviews and summary statements}

Findings of systematic reviews and agreed summary statements reached through the Delphi process are summarised in online supplemental appendix A. Accompanying papers report full methods and results. ${ }^{810-12}$

\section{Survey of expert and experienced clinicians}

Information regarding the study methodology can be accessed in the companion paper. ${ }^{9}$ All statements that reached consensus are summarised in online supplemental appendix B and form the basis of recommendations for clinical practice (see below). The findings of this study represent current clinical expertise.

\section{SUMMARY RECOMMENDATIONS FOR CLINICAL PRACTICE}

We created recommendations for preventing and managing rowingrelated LBP by synthesising the information from research and clinical practice (the assessment and management). The following clinical practice guideline is primarily guided by results from the survey of experienced and expert clinicians as a framework ${ }^{9}$ and embeds information from the Delphi outputs of each work group (Epidemiology, Management, Biomechanics and Athlete voice (qualitative)) throughout.

\section{What is rowing-related low back pain and how prevalent is it?}

A definition of rowing related LBP is summarised in box 2 . An average of $61 \%$ of adult rowers will have experienced an episode of LBP in a 12 -month period. ${ }^{12}$ This compares with a 12 -month prevalence of $51 \%$ in athletes overall ${ }^{12}$ and $37 \%$ in the general population. ${ }^{1}$

\section{What causes rowing-related low back pain, and can it be prevented?}

Risk factors contributing to rowing-related LBP are listed in box 3 .

Factors identified in high-quality rowing-specific studies highlight a history of LBP, rapid increases in training/competition load and ergometer training, particularly sessions lasting longer than $30 \mathrm{~min}$. There were no studies that specifically addressed prevention of rowing-related LBP. In their absence, it is pertinent to modify 


\section{Box 2 Definition of rowing-related LBP}

Low back pain (LBP) is a symptom that can result from several different known or unknown abnormalities or diseases. It is defined by the location of pain, typically between the lower rib margins and the buttock creases. In some cases, it may be accompanied by pain in one or both legs and some people with LBP have associated neurological symptoms in the lower limbs. Rowing-related LBP is pain that affects a rowing athlete, that is because of or exacerbated by rowing or rowing-related training, resulting in a need to modify or stop scheduled activities.

exposure to known risk factors where possible. For example, to monitor response to training load and alter where needed, avoid load spikes and avoid high volumes of ergometer training.

\section{How should rowing-related low back pain be managed?}

General recommendations common to each phase

Physical and non-physical factors contribute to both the onset and persistence of pain. A culture of early recognition and management of LBP should be adopted in the training environment. Most episodes of rowing-related LBP are unlikely to be serious and will likely be self-limiting. Rowers with LBP often report feeling isolated during rehabilitation. Coaches and support teams should create an environment where rowers are educated about the nature and presentation of LBP and supported and encouraged to disclose their LBP early to improve outcomes. Clinicians should have a consistent message with a clinical alliance among each other and a therapeutic alliance with the rower. Ongoing education and reassurance should be provided; it is important to include the rower in decision-making from initial triage to return to on-water training.

Psychological stressors such as poor sleep, performance pressure, fear avoidance behaviour and life stressors signal consideration for psychological support. Other levels of appropriate support may be provided by coaching staff, medical staff, family and friends. Rowers should be encouraged to seek mental health support if there is a specific need. Coaches' and athletes' expectations should be managed. In the elite environment, the coach should be involved from the outset (if the athlete consents) and the coach should be encouraged to share ideas about contributing factors to LBP. An athlete-centred approach should be adopted at all stages. Yellow flags should be considered to minimise fear avoidance behaviour and catastrophising. Rowers should avoid developing a fear of specific movement patterns.

The following are general assessment and management recommendations from initial presentation to return to sport (RTS). Recommendations for each phase with specific details are presented in online supplemental table 3 .

At the time of initial presentation and during the acute phase of recovery, prioritise:

1. Comprehensive assessment for early identification of red and yellow flags.

2. Effective pain control for activities of everyday life.

3. Keeping the rower active with cross training.

4. Regaining rower-specific movement patterns.

5. Empowering and educating the rower and coach.

During the subacute phase and through rehabilitation to full RTS, prioritise:

1. Progressively increasing on-water training volume and intensity with concomitant reduction in cross-training.

2. Multidisciplinary involvement in the RTS plan.
Box 3 Risk factors associated with rowing-related LBP

Risk factors associated with rowing-related low back pain (LBP) (stratified by type of evidence)

Epidemiology research and systematic review

- Previous history of LBP.

- Rapid increase in training or competition load.

- High volume and intensity of training or competition.

- Increased years of exposure to the sport (career length).

- Exposure to ergometer training, specifically sessions $>30 \mathrm{~min}$.

Biomechanics research and systematic review

- Fatigue and poor technique lead to increased lumbar spine flexion and decreased hip range of motion during the rowing stroke (exacerbated during ergometer compared with onwater rowing).

Delphi survey of experienced and expert clinicians

- Training load considerations.

- Steep increase in training load.

- Reduction in load followed by a sharp increase.

- Increase in training volume without adequate recovery.

- Change to training intensity (athlete training outside prescribed or intended training zone).

- Illness or injury prior to LBP episode causing a reduced training load.

- Ability to complete high training volume over a longer period of time (3 months is protective for LBP).

- Young 'training age'.

- On-water rowing considerations.

- Changes in crew increasing load on rower with LBP episode.

- Recent change in boat set up.

- Rough water.

- Increased lumbar flexion range or getting to end of range during rowing.

- Rower: physical and movement considerations.

- Hip flexion less than 130 degrees.

- Reduced hamstring flexibility.

- Reduced knee flexion range.

- Reduced posterior chain and abdominal endurance.

- Motor control of deep squat.

- Control deficits when lifting weights.

- Rower: psychological considerations.

- Fear of pain or movement.

- Worry of having LBP for first time.

- Worry of having a subsequent episode of LBP.

- Selection pressure.

- Increased stress related to being close to a key event.

- Other considerations.

- Poor nutrition or reduced energy intake.

- Poor sleep habits

3. Ensuring modifiable risk factors for rowing-related LBP are addressed.

Criteria for progressing from one phase to the next are shown in figure 2.

Outcome measures and adjunct clinical assessment tools Clinically based outcome measures

Useful outcome measures for assessment at triage and through progression by experienced clinicians are as follows: Visual Analogue Scale; Patient-Specific Functional Scale; Orebro Musculoskeletal 


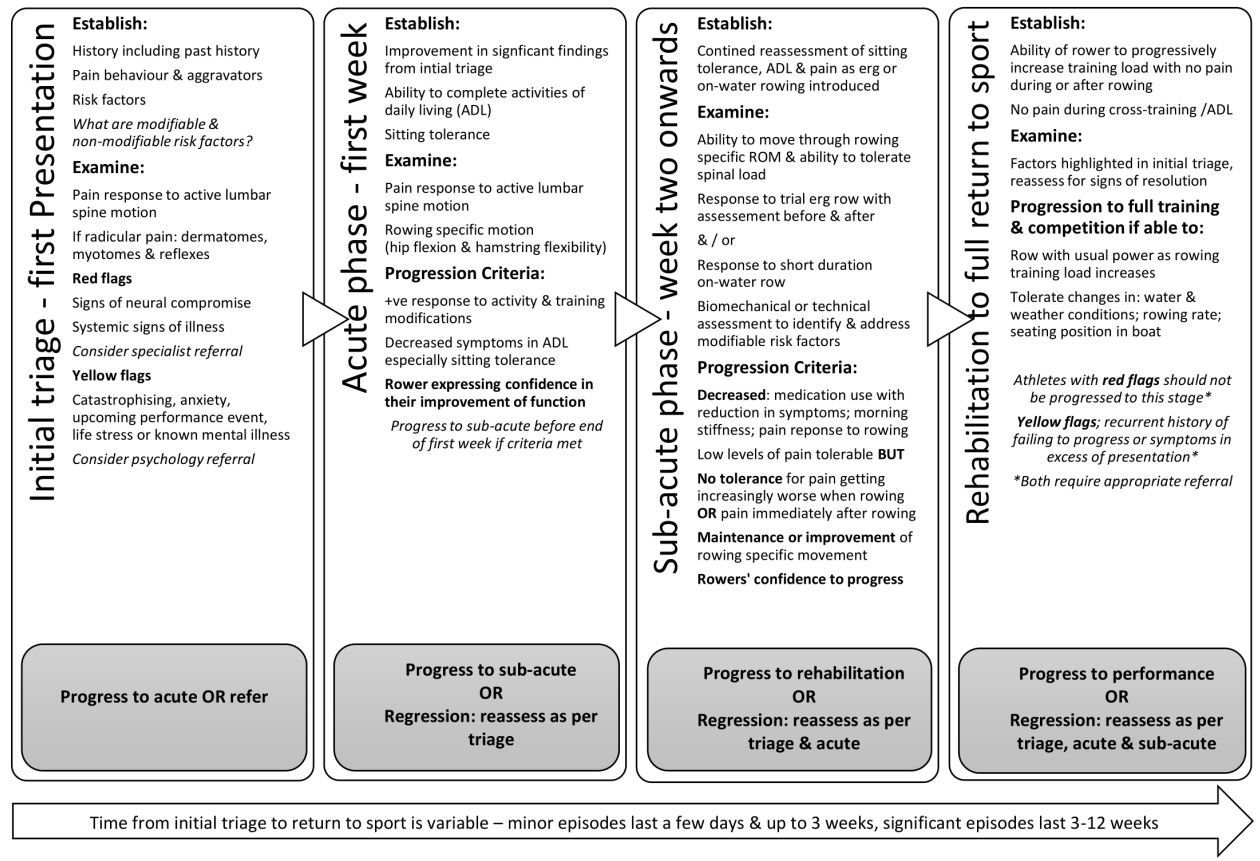

Figure 2 Acute episode of rowing-related low back pain: progression through phases from initial presentation to full return to sport.

Pain Screening Questionnaire and simple, functionally orientated questions, such as the influence of pain on ability to row.

\section{Biomechanics}

The relationship of biomechanics to prevention and treatment of rowing-related LBP is unclear. Tools that have been used in research for monitoring rowing-appropriate biomechanics in this population are as follows: electromagnetic motion measuring devices (eg, Flock of Birds system); two or three-dimensional motion analysis systems (eg, video recording or 3-D motion capture system); ROM devices (eg, goniometer or inclinometer); force measurement devices (eg, load cell attached to the rowing ergometer handle or in the foot plate); electromyography and isokinetic or isometric tests (eg, isokinetic dynamometer). Further research exploring clinical application of these tools is required before recommendations for practice can be made.

\section{Imaging}

There is no evidence to support routine imaging of the low back in rowers. Anatomical MRI changes are common in the lumbar spine of symptomatic ${ }^{14}$ and asymptomatic ${ }^{15}$ rowers. Just as in the general population, interpret MRI findings in a rower in the context of the clinical presentation. Limit imaging to investigating trauma or significant neurology, or where the imaging findings would influence the rower's management plan.

\section{Surgery}

Surgery may be indicated if there are progressive or distinct neurological signs and symptoms, and a clear surgical target. It is unclear whether return to rowing rates are different after surgical management versus after non-surgical management, and we could not identify any randomised controlled trials. Thus, our findings support recent non-systematic reviews on athlete LBP. Reviews focused on surgical outcomes report that surgery is more effective than nonsurgical treatment at reducing pain in the short-term and mediumterm, but these effects do not persist. ${ }^{16} 17$

\section{Pharmacology}

There were no specific recommendations made for managing rowing-related LBP in any studies. Guidelines for pharmacological management of LBP in the general population and for athletes ${ }^{18}$ should be followed according to the World Anti-Doping Agency rules.

\section{Plain language version}

The plain language version of this document is included in the online supplemental file along with the plain language version of online supplemental table 3 (labelled as plain language online supplemental table $3 \mathrm{~A}$ ) and progression flow chart (labelled as plain language online supplemental figure 2A).

\section{DISCUSSION}

In our original meeting in February 2018, we raised a number of questions regarding rowing-related LBP. We wanted to explore the prevalence of rowing-related LBP and how it compared with other sports and LBP in the general population. While the quality and heterogeneity of published studies examining back pain in sports precludes confident comparisons, rowers may have elevated risk of experiencing LBP when compared with the general population, and rowing appears to be a sport with a higher prevalence of LBP than many other sports.

We explored the management of rowing-related LBP through a review of published research and by consulting experts. In general, there was an absence of good quality research examining interventions for LBP in athletes, and we could not find any specifically tailored to rowers. We synthesised available information from our review with recommendations from clinicians (gathered through a survey) to form the framework of a consensus statement to inform clinical practice. Some of these principles are supported by recommendations for managing LBP in the general population but are expanded with advice tailored to the rowing context, particularly in progression through phases to allow RTS. We sought opinions and feedback from rowers 
and coaches and used this feedback to create a plain language summary that was meaningful to end users.

We investigated if rowing-related LBP could be prevented. We identified risk and other factors associated with an episode of rowing-related LBP, but there was no research investigating effectiveness of interventions aimed to prevent LBP in rowers. This suggests that modifying exposures to risk factors where possible may currently be the best approach to preventing LBP in rowers.

Synthesis of evidence from different sources allowed us to create recommendations that were meaningful to rowers and coaches. We highlighted the inadequate research evidence on athlete LBP and call for more quality research. The complexities of LBP were not addressed in athletes; no research has adequately explored the biopsychosocial interactions in rowing-related LBP.

\section{Target populations}

The target user population for this statement is healthcare providers who are managing rowing-related LBP at elite and subelite levels. The plain language summary provides information for adult rowers, coaches and support staff. We intend the recommendations to provide education regarding how best to prevent rowing-related LBP and how to reduce the impact of LBP when it does occur, including how best to avoid the recurrence of pain or persistent pain. The consensus statement applies to rowers with and without LBP, so that rowers and clinicians working with rowers may consider the recommendations in the context of primary and secondary LBP prevention.

The target patient population is adult male and female rowers of all boat and weight classes in all rowing settings from club and college to international standard. When applying the information in the consensus statement to masters rowers, the clinician should consider aspects of normal ageing and age-related disease. The information in the consensus statement may not apply to youth (junior) level rowers who are under 18 years, or para rowers.

\section{Expected outcomes}

The consensus statement provides a framework to inform best care based on current evidence and clinical expertise. It includes end users by highlighting the lived experience of rowers ('rower's voice') from qualitative research and the rowers' and coaches' input into a plain language summary. This document reflects the current state of knowledge and should be read in conjunction with the accompanying systematic reviews and other companion papers, ${ }^{8-12}$ which provide context.

\section{Updating, applicability and dissemination}

We aimed to highlight evidence gaps and create a call for action. A further outcome is a call for action to update the consensus statement as new evidence emerges. It is intended that this consensus statement will be formally reviewed and updated at 5 -year intervals with the first review before 31 December 2025. Barriers to application of this guideline may be resource availability in some settings, including access to a healthcare provider with adequate experience. The plain language summary provides information that may be helpful for athletes without access to such healthcare. Tools that will be used to promote access to this guideline will be open access publishing in the host journal and on the worldrowing.com website. An infographic will promote key messages. Following publication and after a defined period of time, rowing nations will be surveyed to explore their use of the recommendations. To measure dissemination, the core expert group recommended using download metrics of this paper, and the number of engagements on social media when the recommendations are disseminated through the channels described above.

\section{Research priorities}

Prospective studies across diverse rowing populations (age groups, boat/rowing types and ability levels including para rowers) are required to establish incidence of and risk factors for rowing-related LBP. A standard definition of rowing-related LBP should be used and refined as needed. We introduced a definition in our recommendations. High-quality randomised controlled trials are urgently needed to determine the effectiveness of individual interventions and management strategies (particularly surgery) from initial acute care to a full return to training and competition. A care pathway should be created for junior, para and masters rowers.

\section{Limitations}

A limitation of the consensus statement methods was that the members of each working group also participated in the Delphi process for their respective topics. Working group members were blinded to responses where possible, but there is a risk of bias.

\section{CONCLUSIONS}

We present a consensus statement for best practice in rowingrelated LBP. This statement is based on research evidence and clinical practice and aligns with recommendations from international guidelines for managing LBP in the general population, and includes specific recommendations for rowers. Research efforts in relation to all aspects of managing rowing-related and athlete-related LBP should be intensified. It is our hope that the statement will help guide decisions regarding prevention and management of rowers with LBP. Future research should focus on a standardised approach to defining, assessing and managing LBP in rowers, encompassing the biopsychosocial influences on LBP.

\section{Author affiliations}

${ }^{1}$ Discipline of Physiotherapy, Trinity College Dublin School of Medicine, Dublin, Ireland

${ }^{2}$ Family Medicine, Schulich School of Medicine \& Dentistry, University of Western

Ontario, London, Ontario, Canada

${ }^{3}$ Epidemiology \& Biostatistics, Schulich School of Medicine \& Dentistry, University of

Western Ontario, London, Ontario, Canada

${ }^{4}$ Hobart, Tasmania, Australia

${ }^{5}$ Department of Sports Science and Clinical Biomechanics, University of Southern

Denmark, Odense, Syddanmark, Denmark

${ }^{6}$ Copenhagen, Denmark

${ }^{7}$ Division of Sports Medicine- Boston Children's Hospital, Neuroendocrine Unit-

Massachusetts General Hospital, Harvard Medical School, Boston, Massachusetts, USA

${ }^{8}$ School of Physiotherapy and Exercise Science, Curtin University, Perth, Western

Australia, Australia

${ }^{9}$ University of Tasmania School of Medicine, Hobart, Tasmania, Australia

${ }^{10}$ Department of Physical Education and Sports Sciences, University of Limerick,

Limerick, Ireland

${ }^{11}$ Sport Ireland Institute, Abbotstown, Dublin, Ireland

${ }^{12}$ Surgery and Cancer/Human Performance Group, Faculty of Medicine, Imperial College London, London, UK

${ }^{13}$ High Performance Sport New Zealand, Auckland, New Zealand

${ }^{14}$ Sport and Exercise Medicine Research Centre, La Trobe University, Melbourne,

Victoria, Australia

${ }^{15}$ Division of Physiotherapy, Karolinska Institute, Huddinge, Sweden

Correction notice This article has been corrected since it published Online First. The supplemental files and acknowledgements statement have been updated. 
Twitter Fiona Wilson @fionawilsonf, Jane S Thornton @janesthornton, Kathryn E Ackerman @drkateackerman, Larissa Trease @DrLarissaTrease and Clare L Ardern @clare_ardern

Acknowledgements Athlete representatives: Gary O'Donovan (Ireland), Frida Svensson (Sweden), Kim Brennan (Australia). Coach representatives: Mads Haubro (Denmark), Paul Thompson (formerly GB and now China). World Rowing Sports Medicine Committee: Juergen Steinacker, Jo Hannafin, Mike Wilkinson, Mikio Hiura, Piero Poli, Donia Koubaa, Henning Bay Nielsen, Tomislav Smoljanovic, Petra Zupet. Graphic design Figure 1: Ciaran Ward (independent graphic designer). We would like to thank the following people for their contributions at different stages, or within subsections, during the creation of this consensus statement: Ann Redgrave, Mark Edgar, Caroline MacManus, Bruce Forster, Kieran O'Sullivan, Phil Glasgow, Leo Ng, Peter O'Sullivan, Ben Clarsen, Connie Draper, Alex Wolf.

Contributors All authors have made substantial contributions to: (1) the conception and design of the study, or acquisition of data, or analysis and interpretation of data; (2) drafting the article or revising it critically for important intellectual content and (3) final approval of the version submitted.

Funding Trinity College Dublin funded the consensus statement through internal research funding to FW, but did not influence the content of this consensus statement. The Australian Olympic Medical Committee provided funding for KW to attend the first meeting in London. High Performance Sport New Zealand funded CN through the Prime Minister's Scholarship for HPSNZ.

Competing interests None declared.

Patient consent for publication Not required.

Provenance and peer review Not commissioned; externally peer reviewed.

Supplemental material This content has been supplied by the author(s). It has not been vetted by BMJ Publishing Group Limited (BMJ) and may not have been peer-reviewed. Any opinions or recommendations discussed are solely those of the author(s) and are not endorsed by BMJ. BMJ disclaims all liability and responsibility arising from any reliance placed on the content. Where the content includes any translated material, BMJ does not warrant the accuracy and reliability of the translations (including but not limited to local regulations, clinical guidelines, terminology, drug names and drug dosages), and is not responsible for any error and/or omissions arising from translation and adaptation or otherwise.

\section{ORCID iDs}

Fiona Wilson http://orcid.org/0000-0002-0292-1087

Jane S Thornton http://orcid.org/0000-0002-3519-7101

Kathryn E Ackerman http://orcid.org/0000-0003-2626-7785

Larissa Trease http://orcid.org/0000-0002-8413-2814

Clare L Ardern http://orcid.org/0000-0001-8102-3631

\section{REFERENCES}

1 Hartvigsen J, Hancock MJ, Kongsted A, et al. What low back pain is and why we need to pay attention. Lancet 2018:391:2356-67.

2 Kongsted A, Kent P, Axen I, et al. What have we learned from ten years of trajectory research in low back pain? BMC Musculoskelet Disord 2016;17:220.

3 Wilson F, Gissane C, McGregor A. Ergometer training volume and previous injury predict back pain in rowing; strategies for injury prevention and rehabilitation. $\mathrm{Br} J$ Sports Med 2014:48:1534-7.

4 Mäestu J, Jürimäe J, Jürimäe T. Monitoring of performance and training in rowing. Sports Med 2005:35:597-617.

5 Trease L, Wilkie K, Lovell G, et al. Epidemiology of injury and illness in 153 Australian international-level rowers over eight international seasons. Br J Sports Med 2020;54:1288-93.

6 Brouwers MC, Kerkvliet K, Spithoff K, et al. The agree reporting checklist: a tool to improve reporting of clinical practice guidelines. BMJ 2016;352:i1152.

7 Wilson F. Back pain in rowing - update on current understanding: Worldrowing 2016. Available: www.worldrowing.com/news/back-pain-rowing-update-currentunderstanding

8 Thornton JS, Caneiro JP, Hartvigsen J, et al. Treating low back pain in athletes: a systematic review with meta-analysis. Br J Sports Med 2021;55:656-62.

9 Wilkie K, Thornton JS, Vinther A, et al. Clinical management of acute low back pain in elite and subelite rowers: a Delphi study of experienced and expert clinicians. $\mathrm{Br} J$ Sports Med 2021. doi:10.1136/bjsports-2020-102520. [Epub ahead of print: 11 Jan 2021].

10 Wilson F, Ng L, O'Sullivan K, et al. 'You're the best liar in the world': a grounded theory study of rowing athletes' experience of low back pain. Br I Sports Med 2021; 55:327-35.

11 Nugent FJ, Vinther A, McGregor A, et al. The relationship between rowing-related low back pain and rowing biomechanics: a systematic review. Br I Sports Med 2021:55:616-30.

12 Wilson F, Ardern CL, Hartvigsen J, et al. Prevalence and risk factors for back pain in sports: a systematic review with meta-analysis. Br I Sports Med 2021:55:601-7.

13 Chandler J, Churchill R, Higgins J. Methodological expectations of Cochrane intervention reviews (MECIR) standards for the reporting of plain language summaries in new Cochrane intervention reviews, 2013.

14 Hosea T, Hannafin J, Bran J, et al. Aetiology of low back pain in young athletes: role of sport type. Br I Sports Med 2011;45:352-52

15 Maurer M, Soder RB, Baldisserotto M. Spine abnormalities depicted by magnetic resonance imaging in adolescent rowers. Am J Sports Med 2011;39:392-7.

16 Chen B-L, Guo J-B, Zhang H-W, et al. Surgical versus non-operative treatment for lumbar disc herniation: a systematic review and meta-analysis. Clin Rehabil 2018;32:146-60

17 Clark R, Weber RP, Kahwati L. Surgical management of lumbar radiculopathy: a systematic review. J Gen Intern Med 2020;35:855-64.

18 Hainline B, Turner JA, Caneiro JP, et al. Pain in elite athletes-neurophysiological, biomechanical and psychosocial considerations: a narrative review. Br J Sports Med 2017:51:1259-64. 